\title{
Periodic Orbits of the First Kind in the CR4BP When the Second Primary Is a Triaxial Rigid Body
}

\author{
M. R. Hassan1, Payal Singh², Md. Aminul Hassan³ \\ ${ }^{1}$ Department of Mathematics, S. M. College, T. M. Bhagalpur University, Bhagalpur, India \\ ${ }^{2} \mathrm{~T}$. M. Bhagalpur University, Bhagalpur, India \\ ${ }^{3}$ GTE, Bangalore, India \\ Email: hassansmc@gmail.com, mahassan012@gmail.com
}

How to cite this paper: Hassan, M.R., Singh, P. and Hassan, Md.A. (2017) Periodic Orbits of the First Kind in the CR4BP When the Second Primary Is a Triaxial Rigid Body. International Journal of Astronomy and Astrophysics, 7, 133-153.

https://doi.org/10.4236/ijaa.2017.73011

Received: April 3, 2017

Accepted: July 21, 2017

Published: July 24, 2017

Copyright (c) 2017 by authors and Scientific Research Publishing Inc. This work is licensed under the Creative Commons Attribution International License (CC BY 4.0). http://creativecommons.org/licenses/by/4.0/

\begin{abstract}
The present paper deals with the existence of periodic orbits in the Circular Restricted Four-Body Problem (CR4BP) in two-dimensional co-ordinate system when the second primary is a triaxial rigid body and the third primary of inferior mass (in comparison of the other primaries) is placed at triangular libration point $L_{4}$ of the Circular Restricted Three-Body Problem (CR3BP). With the help of generating solutions, we formed a basis for the existence of periodic orbits, then an analytical approach given by Hassan et al. [1], was applied to our model of equilateral triangular configuration. It is found that in general solution also; the character of periodic orbits is conserved. For verification of the existence of periodic orbits, we have applied the criterion of Duboshin [2] and found satisfied.
\end{abstract}

\section{Keywords}

Autonomous Four-Body Problem, CR4BP, Triaxial Rigid Body,

Regularization, Generating and General Solution, Periodicity

\section{Introduction}

Giacaglia [3] applied the method of analytic continuation to examine the existence of periodic orbits of collision of the first kind in the CR3BP. Bhatnagar [4] generalized the problem in elliptic case. Further Bhatnagar [5] extended the work of Giacaglia [3] in the CR4BP by considering three primaries at the vertices of an equilateral triangle. In last three decades, a series of works have been performed by different authors with different perturbations in the circular and elliptic restricted three-body and four-body problem but nobody established the 
proper mathematical model of the Restricted Four-Body Problem (R4BP).

Recently Ceccaroni and Biggs [6] studied the autonomous coplanar CR4BP with an extension to low-thrust propulsion for application to the future science mission. In their problem, they also studied the stability region of the artificial and natural equilibrium points in the Sun-Jupiter Trojan Asteroid-Spacecraft system. Using the concept of Ceccaroni and Biggs [6] and the method of Hassan et al. [1], we have proposed to study the existence of periodic orbits of the first kind in the autonomous R4BP by considering the second primary as a triaxial rigid body.

\section{Equations of Motion of the Infinitesimal Mass}

Let $P_{i}(i=1,2,3)$ be the three primaries of masses $m_{j}(j=1,2,3)$ respectively, where $m_{1} \geq m_{2}>m_{3}$ and the fourth body $P_{4}$ of infinitesimal mass $m$ be assumed so small that it can't influence the motion of the primaries but the motion of $P_{4}(m)$ is influenced by them. Moreover, we assumed that the mass $m_{3}$ (mass of the third primary placed at $L_{4}$ of the R3BP) is small enough so that it can't influence the motion of the two dominating primaries $P_{1}$ and $P_{2}$ but can influence the motion of the infinitesimal body $P_{4}(m)$.

Thus the centre of mass (i.e. the bary-centre) i.e. the centre of rotation of the system remains at the bary-centre $O$ of the two primaries $P_{1}$ and $P_{2}$. Also, all the primaries $P_{1}, P_{2}$ and $P_{3}$ are moving in the same plane of motion in different circular orbits of radii $O P_{1}, O P_{2}$ and $O P_{3}$ respectively around the bary-centre $O$ with the same angular velocity $\omega$. Considering $(O, X Y)$ as an inertial frame in such a way that the $X Y$-plane coincides with the plane of motion of the primaries and origin coincides with $O$. Initially let the principal axes of the second primary $P_{2}$ are parallel to the synodic axes $(O, \xi \eta)$ and its axis of symmetry is perpendicular to the plane of motion. Since the primaries are revolving without rotation about $O$ with the same angular velocity as that of the synodic axes, hence, the principal axes of $P_{2}$ will remain parallel to the co-ordinate axes throughout the motion.

Let at any time $t, P_{1}\left(\xi_{1}, 0\right)$ and $P_{2}\left(\xi_{2}, 0\right)$ be the positions of two dominating primaries on the $x$-axis of the rotating (synodic) co-ordinate system and $P_{3}\left(\xi_{3}, \eta_{3}\right)$ be the third primary placed at the equilibrium point $L_{4}$ of $P_{1}$ and $P_{2}$. Let $\boldsymbol{r}_{1}, \boldsymbol{r}_{2}$ and $\boldsymbol{r}_{3}$ be the displacements of $P_{1}, P_{2}$ and $P_{3}$ relative to $P_{4}$ as shown in Figure 1 and $\boldsymbol{r}$ be the position vector of $P_{4}(x, y)$, then

$$
\begin{aligned}
& \boldsymbol{r}=x \hat{i}+y \hat{j}=\boldsymbol{O P}_{4}, \\
& \boldsymbol{r}_{1}=\left(x-\xi_{1}\right) \hat{i}+y \hat{j}=\boldsymbol{P}_{1} \boldsymbol{P}_{4}, \\
& \boldsymbol{r}_{2}=\left(x-\xi_{2}\right) \hat{i}+y \hat{j}=\boldsymbol{P}_{2} \boldsymbol{P}_{4}, \\
& \boldsymbol{r}_{3}=\left(x-\xi_{3}\right) \hat{i}+\left(y-\eta_{3}\right) \hat{j}=\boldsymbol{P}_{3} \boldsymbol{P}_{4} .
\end{aligned}
$$

Let $\boldsymbol{F}_{1}, \boldsymbol{F}_{2}$ and $\boldsymbol{F}_{3}$ be the gravitational forces exerted by the primaries $P_{1}, P_{2}$ and $P_{3}$ respectively on the infinitesimal mass $m$ at $P_{4}(x, y)$, then

$$
\boldsymbol{F}_{1}=-\frac{\gamma m m_{1}}{r_{1}^{3}}\left\{\left(x-\xi_{1}\right) \hat{i}+y \hat{j}\right\} .
$$

where $\gamma$ is the gravitational constant. 


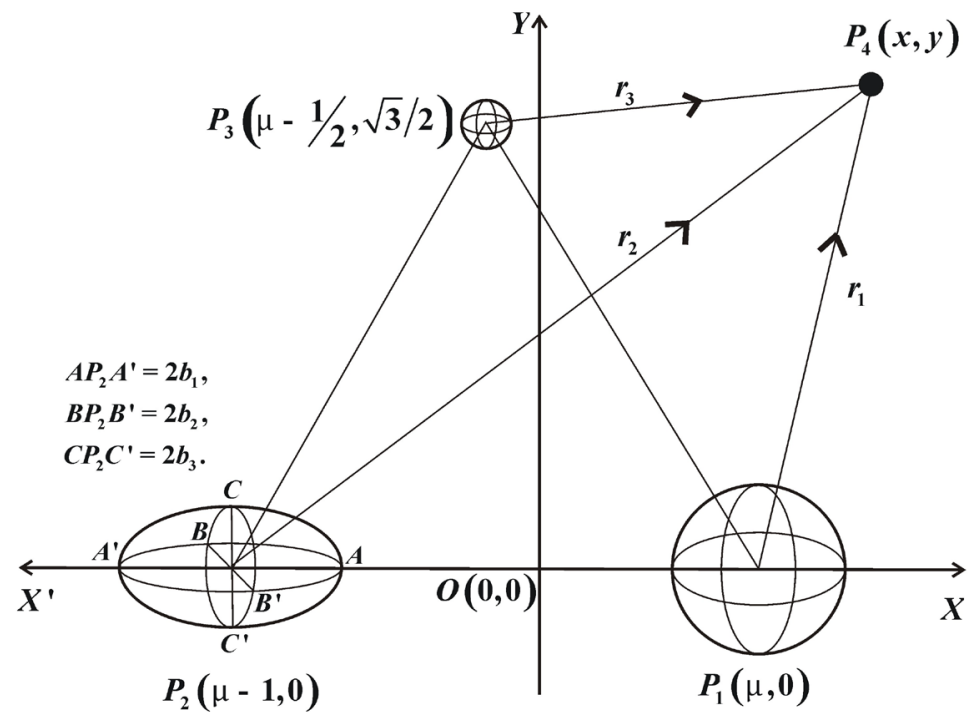

Figure 1. Configuration of CR4BP when the second primary is a Triaxial rigid body.

Let $b_{1}, b_{2}$ and $b_{3}$ be the lengths of the semi-axes of the second primary $P_{2}\left(\xi_{2}, 0\right)$ then the gravitational force exerted by $P_{2}\left(\xi_{2}, 0\right)$ on $P_{4}(x, y)$ is given by McCuskey [7]

$$
\boldsymbol{F}_{2}=-\frac{\gamma m m_{2}}{r_{2}^{3}} \hat{r}_{2}-\frac{3 \gamma m m_{2}}{2 r_{2}^{4}}\left(\frac{2 b_{1}^{2}-b_{2}^{2}-b_{3}^{2}}{5 R^{2}}\right) \hat{r}_{2}+\frac{15 \gamma m m_{2}}{2 r_{2}^{6}}\left(\frac{b_{1}^{2}-b_{2}^{2}}{5 R^{2}}\right) y^{2} \hat{r}_{2} .
$$

Let $\sigma_{1}=\frac{b_{1}^{2}-b_{3}^{2}}{5 R^{2}}, \sigma_{2}=\frac{b_{2}^{2}-b_{3}^{2}}{5 R^{2}}$, then

where $\hat{r}_{2}$ is the unit vector along $\boldsymbol{r}_{2}$ i.e., $\hat{r}_{2}=\frac{\boldsymbol{r}_{2}}{\left|\boldsymbol{r}_{2}\right|}=\frac{\left(x-\xi_{2}\right) \hat{i}+y \hat{j}}{r_{2}}$

$$
\begin{aligned}
\boldsymbol{F}_{2}= & -\gamma m m_{2}\left[\left\{\frac{x-\xi_{2}}{r_{2}^{3}}+\frac{3\left(2 \sigma_{1}-\sigma_{2}\right)\left(x-\xi_{2}\right)}{2 r_{2}^{5}}-\frac{15\left(\sigma_{1}-\sigma_{2}\right)\left(x-\xi_{2}\right)}{2 r_{2}^{7}} y^{2}\right\} \hat{i}\right. \\
& \left.+\left\{\frac{y}{r_{2}^{3}}+\frac{3\left(2 \sigma_{1}-\sigma_{2}\right)}{2 r_{2}^{5}} y-\frac{15\left(\sigma_{1}-\sigma_{2}\right)}{2 r_{2}^{7}} y^{3}\right\} \hat{j}\right]
\end{aligned}
$$

and

$$
\boldsymbol{F}_{3}=-\frac{\gamma m m_{3}}{r_{3}^{3}}\left[\left(x-\xi_{3}\right) \hat{i}+\left(y-\eta_{3}\right) \hat{j}\right] .
$$

Total gravitational force exerted by the three primaries on the infinitesimal mass is given by

$$
\begin{aligned}
\boldsymbol{F}= & \boldsymbol{F}_{1}+\boldsymbol{F}_{2}+\boldsymbol{F}_{3} \\
= & -\gamma m\left[\left\{\frac{m_{1}\left(x-\xi_{1}\right)}{r_{1}^{3}}+\frac{m_{2}\left(x-\xi_{2}\right)}{r_{2}^{3}}+\frac{m_{3}\left(x-\xi_{3}\right)}{r_{3}^{3}}+\frac{3 m_{2}\left(2 \sigma_{1}-\sigma_{2}\right)\left(x-\xi_{2}\right)}{2 r_{2}^{5}}\right.\right. \\
& \left.-\frac{15 m_{2}\left(\sigma_{1}-\sigma_{2}\right)\left(x-\xi_{2}\right)}{2 r_{2}^{7}} y^{2}\right\} \hat{i}+\left\{\frac{m_{1} y}{r_{1}^{3}}+\frac{m_{2} y}{r_{2}^{3}}+\frac{m_{3}\left(y-\eta_{3}\right)}{r_{3}^{3}}+\frac{3 m_{2}\left(2 \sigma_{1}-\sigma_{2}\right)}{2 r_{2}^{5}} y\right. \\
& \left.\left.-\frac{15 m_{2}\left(\sigma_{1}-\sigma_{2}\right)}{2 r_{2}^{7}} y^{3}\right\} \hat{j}\right] .
\end{aligned}
$$


The equation of motion of infinitesimal mass in the gravitational field of the three primaries $P_{1}, P_{2}$ and $P_{3}$ is given by

$$
m\left[\frac{\partial^{2} \boldsymbol{r}}{\partial t^{2}}+2 \boldsymbol{\omega} \times \frac{\partial \boldsymbol{r}}{\partial t}+\frac{\partial \boldsymbol{\omega}}{\partial t} \times \boldsymbol{r}+\boldsymbol{\omega} \times(\boldsymbol{\omega} \times \boldsymbol{r})\right]=\boldsymbol{F},
$$

where

$$
\begin{aligned}
& \frac{\partial^{2} \boldsymbol{r}}{\partial t^{2}}=\ddot{x} \hat{i}+\ddot{y} \hat{j}=\text { relative acceleration, } \\
& \boldsymbol{\omega} \times \frac{\partial \boldsymbol{r}}{\partial t}=n(-\dot{y} \hat{i}+\dot{x} \hat{j})=\text { coriolis acceleration, } \\
& \frac{\partial \boldsymbol{\omega}}{\partial t} \times \boldsymbol{r}=\text { Euler's acceleration, }
\end{aligned}
$$

and $\boldsymbol{\omega} \times(\boldsymbol{\omega} \times \boldsymbol{r})=-n^{2}(x \hat{i}+y \hat{j})=$ centrifugal acceleration,

$$
\text { (as } \omega=n \hat{k} \text { is a constant vector). }
$$

From Equations ((5) and (6)), we get

$$
\begin{gathered}
m\left[\left(\ddot{x}-2 n \dot{y}-n^{2} x\right) \hat{i}+\left(\ddot{y}+2 n \dot{x}-n^{2} y\right) \hat{j}\right]=-\gamma m\left[\left\{\frac{m_{1}\left(x-\xi_{1}\right)}{r_{1}^{3}}+\frac{m_{2}\left(x-\xi_{2}\right)}{r_{2}^{3}}\right.\right. \\
\left.+\frac{m_{3}\left(x-\xi_{3}\right)}{r_{3}^{3}}+\frac{3 m_{2}\left(2 \sigma_{1}-\sigma_{2}\right)\left(x-\xi_{2}\right)}{2 r_{2}^{5}}-\frac{15 m_{2}\left(\sigma_{1}-\sigma_{2}\right)\left(x-\xi_{2}\right)}{2 r_{2}^{7}} y^{2}\right\} \hat{i} \\
\left.+\left\{\frac{m_{1} y}{r_{1}^{3}}+\frac{m_{2} y}{r_{2}^{3}}+\frac{m_{3}\left(y-\eta_{3}\right)}{r_{3}^{3}}+\frac{3 m_{2}\left(2 \sigma_{1}-\sigma_{2}\right)}{2 r_{2}^{5}} y-\frac{15 m_{2}\left(\sigma_{1}-\sigma_{2}\right)}{2 r_{2}^{7}} y^{3}\right\} \hat{j}\right]
\end{gathered}
$$

By equating the coefficients of $\hat{i}$ and $\hat{j}$ from both sides, we get the equations of motion of the infinitesimal mass as

$$
\begin{aligned}
& \ddot{x}-2 n \dot{y}-n^{2} x=-\gamma\left[\frac{m_{1}\left(x-\xi_{1}\right)}{r_{1}^{3}}+\frac{m_{2}\left(x-\xi_{2}\right)}{r_{2}^{3}}+\frac{m_{3}\left(x-\xi_{3}\right)}{r_{3}^{3}}\right. \\
&+\left.\frac{3 m_{2}\left(2 \sigma_{1}-\sigma_{2}\right)\left(x-\xi_{2}\right)}{2 r_{2}^{5}}-\frac{15 m_{2}\left(\sigma_{1}-\sigma_{2}\right)\left(x-\xi_{2}\right)}{2 r_{2}^{7}} y^{2}\right], \\
& \ddot{y}+2 n \dot{x}-n^{2} y=-\gamma\left[\frac{m_{1} y}{r_{1}^{3}}+\frac{m_{2} y}{r_{2}^{3}}+\frac{m_{3}\left(y-\eta_{3}\right)}{r_{3}^{3}}+\frac{3 m_{2}\left(2 \sigma_{1}-\sigma_{2}\right)}{2 r_{2}^{5}} y\right. \\
&\left.-\frac{15 m_{2}\left(\sigma_{1}-\sigma_{2}\right)}{2 r_{2}^{7}} y^{3}\right]
\end{aligned}
$$

Let $\boldsymbol{v}=v_{1} \hat{i}+v_{2} \hat{j}$ be the linear velocity of the infinitesimal mass at $P_{4}(x, y)$ then

$$
\begin{gathered}
\boldsymbol{v}=\frac{\mathrm{d} \boldsymbol{r}}{\mathrm{d} t}=\frac{\partial \boldsymbol{r}}{\partial t}+\boldsymbol{\omega} \times \boldsymbol{r}, \quad\left[\text { as } \frac{\mathrm{d}}{\mathrm{d} t}=\frac{\partial}{\partial t}+\boldsymbol{\omega} \times\right] \\
v_{1} \hat{i}+v_{2} \hat{j}=(\dot{x}-n y) \hat{i}+(\dot{y}+n x) \hat{j}, \\
\Rightarrow v_{1}=\dot{x}-n y, v_{2}=\dot{y}+n x .
\end{gathered}
$$

$\therefore$ Kinetic energy of the infinitesimal mass is given by 
$T=\frac{1}{2}|\boldsymbol{v}|^{2}$ for unit mass of the infinitesimal body.

$$
T=\frac{1}{2}\left(\dot{x}^{2}+\dot{y}^{2}\right)+n(x \dot{y}-\dot{x} y)+\frac{n^{2}}{2}\left(x^{2}+y^{2}\right) .
$$

where the mean motion of the synodic frame is given by

$$
n^{2}=1+\frac{3}{2}\left(2 \sigma_{1}-\sigma_{2}\right)
$$

Let $p_{1}$ and $p_{2}$ be the momenta corresponding to the co-ordinates $x$ and $y$ respectively then $p_{1}=\frac{\partial T}{\partial \dot{x}}, p_{2}=\frac{\partial T}{\partial \dot{y}}$

$$
\Rightarrow p_{1}=\dot{x}-n y=v_{1} \text { and } p_{2}=\dot{y}+n x=v_{2}
$$

Thus

$$
T=\frac{1}{2}\left(p_{1}^{2}+p_{2}^{2}\right)
$$

Let $V_{i}=(i=1,2,3)$ be the gravitational potential of the primaries of masses $m_{i}(i=1,2,3)$ at any point outside of the infinitesimal mass, then

$V_{1}=-\frac{\gamma m_{1}}{r_{1}}, \quad V_{2}=-\frac{\gamma m_{2}}{r_{2}}-\frac{\gamma m_{2}\left(2 \sigma_{1}-\sigma_{2}\right)}{2 r_{2}^{3}}+\frac{3 \gamma m_{2}\left(\sigma_{1}-\sigma_{2}\right)}{2 r_{2}^{5}} y^{2}, \quad V_{3}=-\frac{\gamma m_{3}}{r_{3}}$.

$\therefore$ Total potential at any point outside of the infinitesimal mass due to three primaries is given by

$$
V=\sum_{i=1}^{3} V_{i}=-\gamma\left(\frac{m_{1}}{r_{1}}+\frac{m_{2}}{r_{2}}+\frac{m_{3}}{r_{3}}\right)-\frac{\gamma m_{2}\left(2 \sigma_{1}-\sigma_{2}\right)}{2 r_{2}^{3}}+\frac{3 \gamma m_{2}\left(\sigma_{1}-\sigma_{2}\right)}{2 r_{2}^{5}} y^{2} .
$$

The Hamiltonian of the infinitesimal body of unit mass is given by

$$
\begin{gathered}
H=\sum p \dot{x}-(T-V)=\left(p_{1} \dot{x}+p_{2} \dot{y}\right)-(T-V) \\
H=\frac{1}{2}\left(p_{1}^{2}+p_{2}^{2}\right)+n\left(p_{1} y-p_{2} x\right)-\gamma\left(\frac{m_{1}}{r_{1}}+\frac{m_{2}}{r_{2}}+\frac{m_{3}}{r_{3}}\right)-\frac{\gamma m_{2}\left(2 \sigma_{1}-\sigma_{2}\right)}{2 r_{2}^{3}} \\
+\frac{3 \gamma m_{2}\left(\sigma_{1}-\sigma_{2}\right)}{2 r_{2}^{5}} y^{2}=C=\text { Constant }
\end{gathered}
$$

Assuming $\mu$ as the mass ratio of $m_{2}$ and $\varepsilon$ as the mass ratio of $m_{3}$ to the total mass of the dominating primaries $P_{1}$ and $P_{2}$ then $\mu=\frac{m_{2}}{m_{1}+m_{2}}$ and $\varepsilon=\frac{m_{3}}{m_{1}+m_{2}}$. Also assuming $m_{1}+m_{2}=1$ then $m_{2}=\mu, m_{1}=1-\mu$ and $m_{3}=\varepsilon$. From the definition of the centre of mass of $m_{1}$ and $m_{2}$ we have $m_{1} \xi_{1}+m_{2} \xi_{2}=0$ which implies $\xi_{1}=\mu, \xi_{2}=\mu-1, \quad \xi_{3}=\mu-\frac{1}{2}$ and $\eta_{3}=\frac{\sqrt{3}}{2}$. Thus the co-ordinates of the three primaries $P_{1}, P_{2}$ and $P_{3}$ are $(\mu, 0),(\mu-1,0)$ and $\left(\mu-\frac{1}{2}, \frac{\sqrt{3}}{2}\right)$ respectively, which confirm $\left|\boldsymbol{P}_{1} \boldsymbol{P}_{2}\right|=\left|\boldsymbol{P}_{2} \boldsymbol{P}_{3}\right|=\left|\boldsymbol{P}_{3} \boldsymbol{P}_{1}\right|=1$ i.e., $P_{1} P_{2} P_{3}$ is an equilateral triangle of sides of unit 
length.

Now choosing unit of time in such a way that $\gamma=1$ and taking $x=x_{1}$ and $y=x_{2}$, then the reduced Hamiltonian is given by

$$
\begin{aligned}
H= & \frac{1}{2}\left(p_{1}^{2}+p_{2}^{2}\right)+n\left(p_{1} x_{2}-p_{2} x_{1}\right)-\frac{1-\mu}{r_{1}}-\frac{\mu}{r_{2}}-\frac{\varepsilon}{r_{3}} \\
& -\frac{\mu\left(2 \sigma_{1}-\sigma_{2}\right)}{2 r_{2}^{3}}+\frac{3 \mu\left(\sigma_{1}-\sigma_{2}\right)}{2 r_{2}^{5}} x^{2}=C .
\end{aligned}
$$

The Hamiltonian-canonical equations are

$$
\frac{\mathrm{d} x_{i}}{\mathrm{~d} t}=\frac{\partial H}{\partial p_{i}}, \frac{\mathrm{d} p_{i}}{\mathrm{~d} t}=-\frac{\partial H}{\partial x_{i}} .(i=1,2)
$$

The energy integral of the infinitesimal mass is

$$
\begin{aligned}
& \frac{1}{2}\left(\dot{x}_{1}^{2}+\dot{x}_{2}^{2}\right) \\
& =\frac{1}{2} n^{2}\left(x_{1}^{2}+x_{2}^{2}\right)+\frac{1-\mu}{r_{1}}+\frac{\mu}{r_{2}}+\frac{\varepsilon}{r_{3}}+\frac{\mu\left(2 \sigma_{1}-\sigma_{2}\right)}{2 r_{2}^{3}}-\frac{3 \mu\left(\sigma_{1}-\sigma_{2}\right)}{2 r_{2}^{5}} x_{2}^{2}+C .
\end{aligned}
$$

where $C$ is a constant.

\section{Regularization}

In our Hamiltonian given in Equation (16), there are three singularities $r_{1}=r_{2}=r_{3}=0$, so to examine the existence of periodic orbits around the first primary; we have to eliminate the singularity $r_{1}=0$ from the Hamiltonian in Equation (16). For this, let us define an extended generating function $S$ by

$$
S=\left(\mu+q_{1}^{2}-q_{2}^{2}\right) p_{1}+2 q_{1} q_{2} p_{2}
$$

where $Q_{i}(i=1,2)$ are momenta associated with new co-ordinates $q_{i}(i=1,2)$ and $x_{i}=\frac{\partial S}{\partial p_{i}}, Q_{i}=\frac{\partial S}{\partial q_{i}}$.

Clearly,

$$
\begin{gathered}
x_{1}=\frac{\partial S}{\partial p_{1}}=\mu+q_{1}^{2}-q_{2}^{2}, \quad x_{2}=\frac{\partial S}{\partial p_{2}}=2 q_{1} q_{2} \\
Q_{1}=2\left(p_{1} q_{1}+p_{2} q_{2}\right), \quad Q_{2}=2\left(p_{2} q_{1}-p_{1} q_{2}\right) \\
r_{1}^{2}=\left(x_{1}-\mu\right)^{2}+x_{2}^{2}=\left(q_{1}^{2}-q_{2}^{2}\right)^{2}+4 q_{1}^{2} q_{2}^{2}=\left(q_{1}^{2}+q_{2}^{2}\right)^{2} \\
r_{1}=q_{1}^{2}+q_{2}^{2}, \quad r_{2}^{2}=1+r_{1}^{2}+2\left(q_{1}^{2}-q_{2}^{2}\right)+\left(q_{1}^{2}+q_{2}^{2}\right)^{2}, \\
r_{3}^{2}=1+r_{1}^{2}+\left(q_{1}^{2}-q_{2}^{2}\right)-2 \sqrt{3} q_{1} q_{2}+\left(q_{1}^{2}+q_{2}^{2}\right)^{2} .
\end{gathered}
$$

From Equation (21), we have

$$
\begin{gathered}
p_{1}=\frac{1}{2 r_{1}}\left(Q_{1} q_{1}-Q_{2} q_{2}\right), \quad p_{2}=\frac{1}{2 r_{1}}\left(Q_{1} q_{2}+Q_{2} q_{1}\right), \\
\therefore p_{1}^{2}+p_{2}^{2}=\frac{1}{4 r_{1}}\left(Q_{1}^{2}+Q_{2}^{2}\right),
\end{gathered}
$$




$$
n\left(p_{1} x_{2}-p_{2} x_{1}\right)=\frac{n}{2}\left(Q_{1} q_{2}-Q_{2} q_{1}\right)-\frac{n \mu}{2 r_{1}}\left(Q_{1} q_{2}+Q_{2} q_{1}\right) .
$$

The combination of Equations ((15), (24) and (25)) gives the Hamiltonian $H$ in terms of new variables $q_{i}, Q_{i}(i=1,2)$ as

$$
\begin{aligned}
H= & \frac{1}{8 r_{1}}\left(Q_{1}^{2}+Q_{2}^{2}\right)+\frac{1}{2} n\left(Q_{1} q_{2}-Q_{2} q_{1}\right)-\frac{n \mu}{2 r_{1}}\left(Q_{1} q_{2}+Q_{2} q_{1}\right) \\
& -\frac{1-\mu}{r_{1}}-\frac{\mu}{r_{2}}-\frac{\varepsilon}{r_{3}}-\frac{\mu\left(2 \sigma_{1}-\sigma_{2}\right)}{2 r_{2}^{3}}+\frac{6 \mu\left(\sigma_{1}-\sigma_{2}\right)}{r_{2}^{5}} q_{1}^{2} q_{2}^{2}=C .
\end{aligned}
$$

Let us introduce pseudo time $\tau$ by the equation

$$
\mathrm{d} t=r_{1} \mathrm{~d} \tau \quad(\tau=0 \text { when } t=0)
$$

The Canonical equations of motion corresponding to the regularized Hamiltonian $K$ are given by

$$
\frac{\mathrm{d} q_{i^{\prime}}}{\mathrm{d} \tau}=\frac{\partial K}{\partial Q_{i}}, \quad \frac{\mathrm{d} Q_{i^{\prime}}}{\mathrm{d} \tau}=-\frac{\partial K}{\partial q_{i}}, \quad(i=1,2)
$$

where the regularized Hamiltonian is given by $K=r_{1}(H-C)=0$

$$
\text { i.e., } \begin{aligned}
K= & \frac{1}{8}\left(Q_{1}^{2}+Q_{2}^{2}\right)+\frac{1}{2} n r_{1}\left(Q_{1} q_{2}-Q_{2} q_{1}\right)-\frac{n \mu}{2}\left(Q_{1} q_{2}+Q_{2} q_{1}\right)-(1-\mu) \\
& -\frac{\mu r_{1}}{r_{2}}-\frac{\varepsilon r_{1}}{r_{3}}-\frac{\mu r_{1}\left(2 \sigma_{1}-\sigma_{2}\right)}{2 r_{2}^{3}}+\frac{6 \mu r_{1}\left(\sigma_{1}-\sigma_{2}\right)}{r_{2}^{5}} q_{1}^{2} q_{2}^{2}-r_{1} C=0 .
\end{aligned}
$$

Since $\varepsilon$ is very-very small in comparison of the masses of the dominating primaries hence $\forall \varepsilon \in] 0, \mu\left[\right.$, we can take $\varepsilon=\mu \varepsilon_{0}$ and $C=C_{0}+\mu C_{1}+\mu^{2} C_{2}+\mu^{3} C_{3}+\cdots$.

Let us write $K=K_{0}+\mu K_{1}=0$ then from Equation (29), we have

$$
\begin{gathered}
K_{0}=\frac{1}{8}\left(Q_{1}^{2}+Q_{2}^{2}\right)+\frac{1}{2} r_{1}\left[n\left(Q_{1} q_{2}-Q_{2} q_{1}\right)-2 C_{0}\right]-1=-\lambda(\text { say }) \\
K_{1}=1-\frac{n}{2}\left(Q_{1} q_{2}+Q_{2} q_{1}\right)-r_{1}\left[C_{1}+\frac{1}{r_{2}}+\frac{\varepsilon_{0}}{r_{3}}+\frac{A}{r_{2}^{3}}-\frac{B q_{1}^{2} q_{2}^{2}}{r_{2}^{5}}\right]
\end{gathered}
$$

where

$$
A=\frac{1}{2}\left(2 \sigma_{1}-\sigma_{2}\right), B=3\left(\sigma_{1}-\sigma_{2}\right)
$$

\section{Generating Solution (i.e., Solution When $\mu=0$ )}

For generating solution, we shall choose $K_{0}$ for our Hamiltonian function, so in order to solve the Hamilton-Jacobi equation associated with $K_{0}$, let us write $Q_{i}=\frac{\partial W}{\partial q_{i}}(i=1,2)$ and $1-\lambda=\alpha>0$ arbitrary constant.

Since $t$ is not involved explicitly in $K_{0}$ hence the Hamilton-Jacobi equation may be written as

$$
\frac{1}{8}\left[\left(\frac{\partial W}{\partial q_{1}}\right)^{2}+\left(\frac{\partial W}{\partial q_{2}}\right)^{2}\right]+\frac{1}{2} r_{1}\left[n\left(q_{2} \frac{\partial W}{\partial q_{1}}-q_{1} \frac{\partial W}{\partial q_{2}}\right)-2 C_{0}\right]=\alpha
$$


Putting $q_{1}=\rho \cos \varphi, q_{2}=\rho \sin \varphi$

$$
\rho^{2}=q_{1}^{2}+q_{2}^{2}=r_{1} \text { and } \varphi=\tan ^{-1}\left(\frac{q_{2}}{q_{1}}\right) .
$$

Now $W=W\left(q_{1}, q_{2}\right)=W(\rho, \varphi)$,

$$
\begin{gathered}
\Rightarrow Q_{1}=\frac{\partial W}{\partial q_{1}}=\frac{\partial W}{\partial \rho} \cos \varphi-\frac{\partial W}{\partial \varphi} \cdot \frac{\sin \varphi}{\rho} \\
\text { and } Q_{2}=\frac{\partial W}{\partial q_{2}}=\frac{\partial W}{\partial \rho} \sin \varphi+\frac{\partial W}{\partial \varphi} \cdot \frac{\cos \varphi}{\rho} . \\
\therefore\left(\frac{\partial W}{\partial q_{1}}\right)^{2}+\left(\frac{\partial W}{\partial q_{2}}\right)^{2}=\left(\frac{\partial W}{\partial \rho}\right)^{2}+\frac{1}{\rho^{2}}\left(\frac{\partial W}{\partial \varphi}\right)^{2} \text { and } q_{2} \frac{\partial W}{\partial q_{1}}-q_{1} \frac{\partial W}{\partial q_{2}}=-\frac{\partial W}{\partial \varphi} .
\end{gathered}
$$

Thus the Equation (32) reduces to

$$
\frac{1}{8}\left[\left(\frac{\partial W}{\partial \rho}\right)^{2}+\frac{1}{\rho^{2}}\left(\frac{\partial W}{\partial \varphi}\right)^{2}\right]+\frac{1}{2} \rho^{2}\left[-n \frac{\partial W}{\partial \varphi}-2 C_{0}\right]=\alpha
$$

This is a partial differential equation of second degree, so by the method of variable separable, the solution may be written as

$$
W=U(\rho)+2 G \phi,
$$

where $G$ is an arbitrary constant.

Now introducing a new variable $z$ by $r_{1}=\rho^{2}=z$ then $\frac{\mathrm{d} z}{\mathrm{~d} \rho}=2 \rho$

$$
\begin{gathered}
\therefore \frac{\partial W}{\partial \rho}=\frac{\partial U}{\partial \rho}=\frac{\mathrm{d} U}{\mathrm{~d} \rho}=\frac{\mathrm{d} U}{\mathrm{~d} z} \cdot \frac{\mathrm{d} z}{\mathrm{~d} \rho}=2 \rho \frac{\mathrm{d} U}{\mathrm{~d} z} \\
\text { i.e., } \frac{\partial W}{\partial \rho}=2 \rho \frac{\mathrm{d} U}{\mathrm{~d} z} \text { and } \frac{\partial W}{\partial \varphi}=2 G
\end{gathered}
$$

Introducing Equation (37) in Equation (36), we get

$$
\begin{aligned}
\frac{1}{8}\left[\left(2 \rho \frac{\mathrm{d} U}{\mathrm{~d} z}\right)^{2}+\frac{1}{\rho^{2}}(2 G)^{2}\right]+\frac{1}{2} \rho^{2}\left[-n \cdot 2 G-2 C_{0}\right]=\alpha, \\
z\left(\frac{\mathrm{d} U}{\mathrm{~d} z}\right)^{2}=-\frac{G^{2}}{z}+2 z\left[n \cdot G+C_{0}\right]+2 \alpha, \\
\left(\frac{\mathrm{d} U}{\mathrm{~d} z}\right)^{2}=-\frac{G^{2}}{z^{2}}+2\left[n \cdot G+C_{0}\right]+\frac{2 \alpha}{z}, \\
\quad=-\frac{2\left(n G+C_{0}\right)}{z^{2}}\left[-z^{2}-\frac{\alpha z}{n G+C_{0}}+\frac{G^{2}}{2\left(n G+C_{0}\right)}\right], \\
=-\frac{2\left(n G+C_{0}\right)}{z^{2}} F(z),
\end{aligned}
$$

where

$$
F(z)=-z^{2}-\frac{\alpha z}{n G+C_{0}}+\frac{G^{2}}{2\left(n G+C_{0}\right)} \text { is a quadratic expression in } z .
$$


Thus

$$
\begin{gathered}
\frac{\mathrm{d} U}{\mathrm{~d} z}=\sqrt{-2\left(n G+C_{0}\right)} \frac{\sqrt{F(z)}}{z}, \\
\text { i.e., } U(z, G, \alpha)=\sqrt{-2\left(n G+C_{0}\right)} \int_{z_{1}}^{z} \frac{\sqrt{F(z)}}{z} \mathrm{~d} z,
\end{gathered}
$$

where $z_{1}$ is the smaller root of the equation $F(z)=0$.

From Equation (40), we conclude that for general solution, we need only two arbitrary constants assigned as $\alpha$ and $G$. Therefore the solution (40) may be regarded as a general solution. Following Giacaglia [3] and Bhatnagar [5], let us introduce the parameters $n, a, e, l$ by the relations

$$
z_{1}=n a(1-e), z_{2}=n a(1+e) \text { and } z=z_{1} \cos ^{2} \frac{l}{2}+z_{2} \sin ^{2} \frac{l}{2}=n a(1-e \cos l) .
$$

where $z_{1}$ and $z_{2}$ are the two roots of the equation $F(z)=0, a$ is the semi-major axis, $e$ is the eccentricity and $l$ is the semi-latus rectum of the elliptic orbit of the infinitesimal mass around the first primary. It may be noted that for $z=z_{1}, l=0$.

From Equation (41),

$$
z_{1}+z_{2}=2 n a, \quad z_{1} z_{2}=n^{2} a^{2}\left(1-e^{2}\right)
$$

Again since $z_{1}$ and $z_{2}$ are the roots of the equation $F(z)=0$, hence we have,

$$
z_{1}+z_{2}=-\frac{\alpha}{n G+C_{0}} \text { and } z_{1} z_{2}=-\frac{G^{2}}{2\left(n G+C_{0}\right)} .
$$

From Equations ((42) and (43)),

$$
\begin{aligned}
& 2 n a=-\frac{\alpha}{n G+C_{0}}, \quad n^{2} a^{2}\left(1-e^{2}\right)=-\frac{G^{2}}{2\left(n G+C_{0}\right)}, \\
& \Rightarrow a=-\frac{\alpha}{2 n\left(n G+C_{0}\right)}=\frac{\alpha}{n\left[-2\left(n G+C_{0}\right)\right]} .
\end{aligned}
$$

Introducing a new parameter $L$ by the relation

$$
\alpha=L\left[-2\left(n G+C_{0}\right)\right]^{\frac{1}{2}}>0
$$

Then

$$
a=\frac{L}{n\left[-2\left(n G+C_{0}\right)\right]^{\frac{1}{2}}}>0
$$

$$
\begin{aligned}
& \text { Also } n^{2} a^{2}\left(1-e^{2}\right)=-\frac{G^{2}}{2\left(n G+C_{0}\right)} \\
& n^{2}\left(1-e^{2}\right) \frac{L^{2}}{n^{2}\left[-2\left(n G+C_{0}\right)\right]}=\frac{G^{2}}{\left[-2\left(n G+C_{0}\right)\right]}
\end{aligned}
$$




$$
\Rightarrow e^{2}=\left(1-\frac{G^{2}}{L^{2}}\right)^{\frac{1}{2}} \leq 1
$$

From Equation (38),

$$
\begin{aligned}
& F(z)=-z^{2}-\frac{\alpha z}{\left(n G+C_{0}\right)}+\frac{G^{2}}{2\left(n G+C_{0}\right)}, \\
& =-z^{2}+\frac{2 z L\left[-2\left(n G+C_{0}\right)\right]^{\frac{1}{2}}}{\left[-2\left(n G+C_{0}\right)\right]}+\frac{G^{2}}{2\left(n G+C_{0}\right)}, \\
& =-z^{2}+2 z \frac{L}{\left[-2\left(n G+C_{0}\right)\right]^{\frac{1}{2}}}+\frac{G^{2}}{2\left(n G+C_{0}\right)}, \quad \text { [using Equation (44)] } \\
& =-z^{2}+2 z a n-z_{1} z_{2}, \\
& =n^{2} a^{2} e^{2}-(z-n a)^{2}, \\
& F(z)=n^{2} a^{2} e^{2} \sin ^{2} l \text {, } \\
& \text { i.e., } \quad F(z)=-z^{2}-\frac{\alpha z}{n\left(G+C_{0}\right)}+\frac{G^{2}}{2\left(n G+C_{0}\right)}=n^{2} a^{2} e^{2} \sin ^{2} l
\end{aligned}
$$

The Hamilton-Canonical equation of motion corresponding to the Hamiltonian $K_{0}$ are given by

$$
\begin{array}{ll}
\frac{\mathrm{d} q_{1}}{\mathrm{~d} \tau}=\frac{\partial K_{0}}{\partial Q_{1}}, & \frac{\mathrm{d} q_{2}}{\mathrm{~d} \tau}=\frac{\partial K_{0}}{\partial Q_{2}}, \\
\frac{\mathrm{d} Q_{1}}{\mathrm{~d} \tau}=-\frac{\partial K_{0}}{\partial q_{1}}, & \frac{\mathrm{d} Q_{2}}{\mathrm{~d} \tau}=-\frac{\partial K_{0}}{\partial q_{2}},
\end{array}
$$

where $K_{0}=\frac{1}{8}\left(Q_{1}^{2}+Q_{2}^{2}\right)+\frac{1}{2} \rho^{2}\left[n\left(Q_{1} q_{2}-Q_{2} q_{1}\right)-2 C_{0}\right]-1$.

$$
\Rightarrow \frac{\partial K_{0}}{\partial Q_{1}}=\frac{1}{4} Q_{1}+\frac{1}{2} \rho^{2} n q_{2}, \quad \frac{\partial K_{0}}{\partial Q_{2}}=\frac{1}{4} Q_{2}-\frac{1}{2} \rho^{2} n q_{1} .
$$

Thus

$$
q_{1}^{\prime}=\frac{1}{4} Q_{1}+\frac{1}{2} \rho^{2} n q_{2} \text { and } q_{2}^{\prime}=\frac{1}{4} Q_{2}-\frac{1}{2} \rho^{2} n q_{1} .
$$

where (') primes denote the differentiation with respect to $\tau$.

Now $\rho^{2}=q_{1}^{2}+q_{2}^{2}=z$

$$
\begin{gathered}
\Rightarrow 2 \rho \frac{\mathrm{d} \rho}{\mathrm{d} \tau}=2 q_{1} \frac{\mathrm{d} q_{1}}{\mathrm{~d} \tau}+2 q_{2} \frac{\mathrm{d} q_{2}}{\mathrm{~d} \tau}=\frac{\mathrm{d} z}{\mathrm{~d} \tau}, \\
\Rightarrow 2 \rho \rho^{\prime}=2\left(q_{1} q_{1}^{\prime}+q_{2} q_{2}^{\prime}\right)=\frac{\mathrm{d} z}{\mathrm{~d} \tau} .
\end{gathered}
$$

But

$$
\begin{aligned}
q_{1} q_{1}^{\prime}+q_{2} q_{2}^{\prime} & =q_{1}\left(\frac{1}{4} Q_{1}+\frac{1}{2} \rho^{2} n q_{2}\right)+q_{2}\left(\frac{1}{4} Q_{2}-\frac{1}{2} \rho^{2} n q_{1}\right) \text { [using Equation (49)] } \\
& =\frac{1}{4}\left(q_{1} Q_{1}+q_{2} Q_{2}\right)
\end{aligned}
$$

Thus 


$$
2 \rho \rho^{\prime}=2 \sum_{i=1}^{2} q_{i} q_{i}^{\prime}=\frac{1}{2} \sum_{i=1}^{2} q_{i} Q_{i}=\frac{\mathrm{d} z}{\mathrm{~d} \tau}
$$

Also

$$
\begin{array}{rlrl}
\sum_{i=1}^{2} q_{i} Q_{i} & =q_{1} Q_{1}+q_{2} Q_{2} \\
& =q_{1}\left(\frac{\partial W}{\partial q_{1}}\right)+q_{2}\left(\frac{\partial W}{\partial q_{2}}\right) & \\
& =\rho \cos \varphi\left(\cos \varphi \frac{\partial W}{\partial \rho}-\frac{\sin \varphi}{\rho} \frac{\partial W}{\partial \varphi}\right)+\rho \sin \varphi\left(\sin \varphi \frac{\partial W}{\partial \rho}+\frac{\cos \varphi}{\rho} \frac{\partial W}{\partial \varphi}\right) \\
& =\rho \frac{\partial W}{\partial \rho} & \text { [using Equation (34)] } \\
& =2 \rho^{2} \frac{\mathrm{d} U}{\mathrm{~d} z} \quad \Rightarrow \sum_{i=1}^{2} q_{i} Q_{i}=2 z\left(\frac{\mathrm{d} U}{\mathrm{~d} z}\right) &
\end{array}
$$

Also from Equations ((39), (51) and (52)),

$\frac{1}{2} \rho \frac{\partial W}{\partial \rho}=z \rho \rho^{\prime}=2 \sum_{i=1}^{2} q_{i} q_{i}^{\prime}=\frac{1}{2} \sum_{i=1}^{2} q_{i} Q_{i}=z \frac{\mathrm{d} U}{\mathrm{~d} z}=\sqrt{-2\left(n G+C_{0}\right) F(z)}=\frac{\mathrm{d} z}{\mathrm{~d} \tau}$

From the last relation of Equation (53), we have

$$
\begin{gathered}
\Rightarrow \frac{\mathrm{d} z}{\mathrm{~d} \tau}=\sqrt{-2\left(n G+C_{0}\right)} \sqrt{F(z)} \\
\Rightarrow \frac{\mathrm{d} z}{\sqrt{F(z)}}=\sqrt{-2\left(n G+C_{0}\right)} \mathrm{d} \tau \\
\Rightarrow \int_{z_{1}}^{z} \frac{\mathrm{d} z}{\sqrt{F(z)}}=\sqrt{-2\left(n G+C_{0}\right)} \int_{\tau_{0}}^{\tau} \mathrm{d} \tau \quad \text { where } \tau=z_{1} \Rightarrow l=0, z=\tau_{0} \\
\Rightarrow \int_{0}^{l} \frac{n a l \sin l \mathrm{~d} l}{n a l \sin l}=\sqrt{-2\left(n G+C_{0}\right)}\left(\tau-\tau_{0}\right) \quad \text { [using Equations (41) and (48)] } \\
\Rightarrow l=\left[-2\left(n G+C_{0}\right)\right]^{\frac{1}{2}}\left(\tau-\tau_{0}\right) \\
\Rightarrow l=\int_{z_{1}}^{z} \frac{\mathrm{d} z}{\sqrt{F(z)}}=\left[-2\left(n G+C_{0}\right)\right]^{\frac{1}{2}}\left(\tau-\tau_{0}\right)
\end{gathered}
$$

Again from Equation (53),

$$
\begin{aligned}
& \frac{\mathrm{d} z}{\mathrm{~d} t} \cdot \frac{\mathrm{d} t}{\mathrm{~d} \tau}=\sqrt{-2\left(n G+C_{0}\right)} \sqrt{F(z)} \\
& \frac{\mathrm{d} z}{\mathrm{~d} t} r_{1}=\sqrt{-2\left(n G+C_{0}\right)} \sqrt{F(z)} \\
& \Rightarrow z \frac{\mathrm{d} z}{\mathrm{~d} t}=\sqrt{-2\left(n G+C_{0}\right)} \sqrt{F(z)} \\
& \Rightarrow \mathrm{d} t=\frac{1}{\left[-2\left(n G+C_{0}\right)\right]^{\frac{1}{2}}} \frac{z \mathrm{~d} z}{\sqrt{F(z)}}
\end{aligned}
$$




$$
\begin{gathered}
\Rightarrow \int_{t_{0}}^{t} \mathrm{~d} t=\frac{1}{\left[-2\left(n G+C_{0}\right)\right]^{\frac{1}{2}}} \int_{0}^{l} \frac{\operatorname{an}(1-e \cos l) \text { ane } \sin l \mathrm{~d} l}{\text { anesin } l} \\
t-t_{0}=\frac{a n}{\left[-2\left(n G+C_{0}\right)\right]^{\frac{1}{2}}}(l-e \sin l),
\end{gathered}
$$

where $t_{0}$ is a constant.

Now taking $L$ and $G$ as arbitrary constants in lieu of $\alpha$ and $G$ and the solutions may be given by the relations

$$
\frac{\partial W}{\partial L}=\frac{\partial U}{\partial L}=\int_{z_{1}}^{z} \frac{\mathrm{d} z}{\sqrt{F(z)}}=l, \quad \frac{\partial W}{\partial G}=\frac{\partial U}{\partial G}+2 \varphi=g .
$$

From Equation (40),

$$
U(z, G, L)=\left[-2\left(n G+C_{0}\right)\right]^{\frac{1}{2}} \int_{z_{1}}^{z} \sqrt{F(z)} \frac{\mathrm{d} z}{z} .
$$

Differentiating partially with respect to $G$, we get

$$
\begin{aligned}
& \frac{\partial U}{\partial G}=\frac{\partial}{\partial G} \int_{z_{1}}^{z} \sqrt{-2\left(n G+C_{0}\right) F(z)} \frac{d z}{z}, \\
& =\int_{z_{1}}^{z} \frac{\partial}{\partial G} \sqrt{-2\left(n G+C_{0}\right) F(z)} \frac{\mathrm{d} z}{z}, \\
& =\int_{z_{1}}^{z} \frac{1}{2 \sqrt{-2\left(n G+C_{0}\right) F(z)}} \frac{\partial}{\partial G}\left[-2\left(n G+C_{0}\right) F(z)\right] \frac{\mathrm{d} z}{z}, \\
& =\int_{z_{1}}^{z} \frac{1}{2 \sqrt{-2\left(n G+C_{0}\right) F(z)}} \frac{\partial}{\partial G}\left[2 z^{2}\left(n G+C_{0}\right)+2 z L\left\{-2\left(n G+C_{0}\right)\right\}^{\frac{1}{2}}-G^{2}\right] \frac{\mathrm{d} z}{z}, \\
& =\int_{z_{1}}^{z} \frac{1}{2 \sqrt{-2\left(n G+C_{0}\right) F(z)}}\left[n z^{2}-n^{2} a z-G\right] \frac{\mathrm{d} z}{z}, \\
& =\frac{1}{\left[-2\left(n G+C_{0}\right)\right]^{\frac{1}{2}}}\left[n \int_{z_{1}}^{z} \frac{z \mathrm{~d} z}{\sqrt{F(z)}}-n^{2} a \int_{z_{1}}^{z} \frac{\mathrm{d} z}{\sqrt{F(z)}}-G \int_{z_{1}}^{z} \frac{\mathrm{d} z}{z \sqrt{F(z)}}\right] \text {, } \\
& =\frac{1}{\left[-2\left(n G+C_{0}\right)\right]^{\frac{1}{2}}}\left[n^{2} a(l-e \sin l)-n^{2} a l-\frac{G}{a n} \int_{0}^{l} \frac{\mathrm{d} l}{(1-e \cos l)}\right] \text {, } \\
& =-\frac{n^{2} a e \sin l}{\left[-2\left(n G+C_{0}\right)\right]^{\frac{1}{2}}}-\frac{G}{n a\left[-2\left(n G+C_{0}\right)\right]^{\frac{1}{2}}} \int_{0}^{l} \frac{\mathrm{d} l}{(1-e \cos l)}, \\
& =-n \frac{L}{\left[-2\left(n G+C_{0}\right)\right]^{\frac{1}{2}}} \frac{e \sin l}{\left[-2\left(n G+C_{0}\right)\right]^{\frac{1}{2}}}-\frac{G}{L} \int_{0}^{l} \frac{\mathrm{d} l}{(1-e \cos l)}, \quad \text { using Equation (45)] } \\
& =\frac{-n L \sin l \sqrt{1-\frac{G^{2}}{L^{2}}}}{-2\left(n G+C_{0}\right)}-\sqrt{1-e^{2}} \int_{0}^{l} \frac{\mathrm{d} l}{(1-e \cos l)}, \\
& =\frac{n \sqrt{L^{2}-G^{2}} \sin l}{2\left(n G+C_{0}\right)}-\sqrt{1-e^{2}} \int_{0}^{l} \frac{\mathrm{d} l}{(1-e \cos l)},
\end{aligned}
$$




$$
\Rightarrow \frac{\partial U}{\partial G}=\frac{n \sqrt{L^{2}-G^{2}} \sin l}{2\left(n G+C_{0}\right)}-h,
$$

where

$$
h=\sqrt{1-e^{2}} \int_{0}^{l} \frac{\mathrm{d} l}{(1-e \cos l)} \quad(e \neq 1) .
$$

From Equation (56),

$$
\begin{gathered}
g=\frac{\partial U}{\partial G}+2 \varphi, \\
\Rightarrow g=2 \varphi+\frac{n \sqrt{L^{2}-G^{2} \sin l}}{2\left(n G+C_{0}\right)}-h, \\
\Rightarrow \varphi=\frac{1}{2}(g+h)-\frac{n \sqrt{L^{2}-G^{2}}}{4\left(n G+C_{0}\right)} \sin l, \quad \text { where }(e \neq 1, G \neq 0, h \neq 0) \\
\text { and } \varphi=\frac{1}{2} g-\frac{n L}{4 C_{0}} \sin l, \quad \text { where }(e=1, G=0, h=0)
\end{gathered}
$$

Now let us find the value of $K_{0}$ in terms of $l, g, L, G$. For this, we have

$$
\begin{aligned}
K_{0} & =\frac{1}{8}\left(Q_{1}^{2}+Q_{2}^{2}\right)+\frac{1}{2} \rho^{2}\left[n\left(Q_{1} q_{2}-Q_{2} q_{1}\right)-2 C_{0}\right]-1, \\
& =\frac{1}{8}\left[\left(\frac{\partial W}{\partial \rho}\right)^{2}+\left(\frac{\partial W}{\partial \varphi}\right)^{2}\right]+\frac{1}{2} \rho^{2}\left[-n \frac{\partial W}{\partial \varphi}-2 C_{0}\right]-1 \\
& =\frac{1}{8}\left[\left(2 \rho \frac{\mathrm{d} U}{\mathrm{~d} z}\right)^{2}+\frac{(2 G)^{2}}{\rho^{2}}\right]+\frac{1}{2} \rho^{2}\left[-2 G n-2 C_{0}\right]-1, \\
& =\frac{1}{2 z}\left[\left(z \frac{\mathrm{d} U}{\mathrm{~d} z}\right)^{2}+G^{2}\right]-z\left(n G+C_{0}\right)-1, \\
& =\frac{1}{2 z}\left[-2\left(n G+C_{0}\right) F(z)+G^{2}\right]-z\left(n G+C_{0}\right)-1, \quad \text { [using Equation (53)] } \\
& =\frac{1}{2 z}\left[2 z^{2}\left(n G+C_{0}\right)+2 \alpha z-G^{2}+G^{2}\right]-z\left(n G+C_{0}\right)-1, \quad \text { [using Equation (38)] } \\
& =z\left(n G+C_{0}\right)+\alpha-z\left(n G+C_{0}\right)-1, \\
& =\alpha-1, \quad \Rightarrow K_{0}=L\left[-2\left(n G+C_{0}\right)\right]^{\frac{1}{2}}-1 .
\end{aligned}
$$

Therefore, for the problem generated by the Hamiltonian $K_{0}$, the equations of motion are

$$
\begin{aligned}
& \frac{\mathrm{d} L}{\mathrm{~d} \tau}=\frac{\partial K_{0}}{\partial l}=0 \Rightarrow L=\text { constant }=L_{0}, \\
& \frac{\mathrm{d} G}{\mathrm{~d} \tau}=\frac{\partial K_{0}}{\partial g}=0 \Rightarrow G=\text { constant }=G_{0}, \\
& \frac{\mathrm{d} l}{\mathrm{~d} \tau}=-\frac{\partial K_{0}}{\partial L}=\left[-2\left(n G+C_{0}\right)\right]^{\frac{1}{2}}=\eta_{l}(\text { say }) \quad \Rightarrow l=\eta_{l} \tau+l_{0}, \\
& \frac{\mathrm{d} g}{\mathrm{~d} \tau}=-\frac{\partial K_{0}}{\partial G}=-\frac{L}{\left[-2\left(n G+C_{0}\right)\right]^{\frac{1}{2}}}=\eta_{g}(\text { say }) \quad \Rightarrow g=\eta_{g} \tau+g_{0} .
\end{aligned}
$$


Further we are to express $q_{i}$ and $Q_{i}(i=1,2)$ in terms of canonical elements $l, g, L, G$.

From Equation (34),

$$
\begin{aligned}
& Q_{1}=\frac{\partial W}{\partial q_{i}}=\cos \varphi \frac{\partial W}{\partial \rho}-\frac{\sin \varphi}{\rho} \frac{\partial W}{\partial \varphi} \\
&= \cos \varphi 2 \rho \frac{\mathrm{d} U}{\mathrm{~d} z}-\frac{\sin \varphi}{\rho} \frac{\partial W}{\partial \varphi} \\
&= \pm \frac{2}{\sqrt{z}}\left[\left\{-2\left(n G+C_{0}\right)\right\}^{\frac{1}{2}} \sqrt{F(z)} \cos \varphi-G \sin \varphi\right], \\
& \text { i.e., } Q_{1}=2\left[\frac{e L \sin l \cos \varphi-G \sin \varphi}{ \pm \sqrt{n a(1-e \cos l)}}\right] .
\end{aligned}
$$

Thus,

$$
\begin{array}{ll}
Q_{1}= \pm \frac{2[e L \sin l \cos \varphi-G \sin \varphi]}{\sqrt{n a(1-e \cos l)}}, & Q_{2}= \pm \frac{2[e L \sin l \cos \varphi+G \sin \varphi]}{\sqrt{n a(1-e \cos l)}}, \\
q_{1}= \pm[n a(1-e \cos l)]^{\frac{1}{2}} \cos \varphi, & q_{2}= \pm[n a(1-e \cos l)]^{\frac{1}{2}} \sin \varphi,
\end{array}
$$

where $\varphi$ is given by the first equation of system (59).

When $e=1, G=0, h=0$, then the variables $q_{i}, Q_{i}(i=1,2)$ can be expressed in terms of canonical elements $(l, g, L, G)$ as

$$
\begin{array}{ll}
Q_{1}= \pm \frac{4 L}{\sqrt{2 a n}} \cos \frac{l}{2} \cos \varphi, & Q_{2}= \pm \frac{4 L}{\sqrt{2 a n}} \cos \frac{l}{2} \sin \varphi, \\
q_{1}= \pm \sqrt{2 a n} \sin \frac{l}{2} \cos \varphi, & q_{2}= \pm \sqrt{2 a n} \sin \frac{l}{2} \sin \varphi,
\end{array}
$$

where $\varphi$ is given by the second equation of (59).

The original synodic cartesian co-ordinates in a uniformly rotating (synodic) system are obtained from the Equations ((20) and (23)) when $\mu=0$, as

$$
\begin{array}{ll}
x_{1}=q_{1}^{2}-q_{2}^{2}, & x_{2}=2 q_{1} q_{2}, \\
p_{1}=\frac{1}{2 z}\left(Q_{1} q_{1}-Q_{2} q_{2}\right), & p_{2}=\frac{1}{2 z}\left(Q_{2} q_{1}-Q_{1} q_{2}\right) .
\end{array}
$$

The sidereal cartesian co-ordinates are obtained by considering the transformation

$$
\begin{aligned}
& X_{1}=x_{1} \cos n t-x_{2} \sin n t, \quad X_{2}=x_{1} \sin n t+x_{2} \cos n t, \\
& \dot{X}_{1}=p_{1} \cos n t-p_{2} \sin n t, \quad \dot{X}_{2}=p_{1} \sin n t+p_{2} \cos n t,
\end{aligned}
$$

where $t$ is given by the Equation (55).

Now let us express $K_{1}$ in terms of the canonical elements $l, g, L, G$.

From Equation (31),

$$
K_{1}=1-\frac{1}{2} n\left(Q_{1} q_{2}+Q_{2} q_{1}\right)-r_{1}\left[C_{1}+\frac{1}{r_{2}}+\frac{\varepsilon_{0}}{r_{3}}+\frac{A}{r_{2}^{3}}-\frac{B q_{1}^{2} q_{2}^{2}}{r_{2}^{5}}\right]
$$

Now, 


$$
\begin{aligned}
Q_{1} q_{2}+Q_{2} q_{1}= & \rho \sin \varphi \frac{\partial W}{\partial q_{1}}+\rho \cos \varphi \frac{\partial W}{\partial q_{2}} \\
& =\rho \sin \varphi\left[\cos \varphi \frac{\partial W}{\partial \rho}-\frac{\sin \varphi}{\rho} \frac{\partial W}{\partial \varphi}\right]+\rho \cos \varphi\left[\sin \varphi \frac{\partial W}{\partial \rho}+\frac{\cos \varphi}{\rho} \frac{\partial W}{\partial \varphi}\right] \\
& =\rho \frac{\partial W}{\partial \rho} \sin 2 \varphi+\frac{\partial W}{\partial \varphi} \cos 2 \varphi \quad[\text { using Equation (34)] } \\
& =2 \sqrt{-2\left(n G+C_{0}\right) F(z)} \sin 2 \varphi+2 G \cos 2 \varphi \quad \text { [using Equation (53)] } \\
& =2 a n e\left[-2\left(n G+C_{0}\right)\right]^{\frac{1}{2}} e \sin l \sin 2 \varphi+2 G \cos \varphi \quad \text { [using Equation (47)] } \\
= & 2 e L \sin l \sin 2 \varphi+2 G \cos 2 \varphi \\
& Q_{1} q_{2}+Q_{2} q_{1}=2[e L \sin l \sin 2 \varphi+G \cos 2 \varphi] \\
& \Rightarrow \frac{n}{2}\left(Q_{1} q_{2}+Q_{2} q_{1}\right)=n[e L \sin l \sin 2 \varphi+2 G \cos 2 \varphi] \\
& q_{1}^{2} q_{2}^{2}=\rho^{2} \cos ^{2} \varphi \rho^{2} \sin ^{2} \varphi=\rho^{4}(\sin \varphi \cos \varphi)^{2}=\frac{z^{2}}{4} \sin ^{2} 2 \varphi
\end{aligned}
$$

Thus,

$$
K_{1}=1-n(e L \sin l \sin 2 \varphi+G \cos 2 \varphi)-z\left[C_{1}+\frac{1}{r_{2}}+\frac{\varepsilon_{0}}{r_{3}}+\frac{A}{r_{2}^{3}}-\frac{B z^{2} \sin ^{2} 2 \varphi}{4 r_{2}^{5}}\right]
$$

where

$$
\begin{aligned}
& r_{1}=n a(1-e \cos l)=z, \\
& r_{2}^{2}=1+z^{2}+2 z \cos 2 \varphi, \\
& r_{3}^{2}=1+z^{2}+z \cos 2 \varphi-\sqrt{3} z \sin 2 \varphi
\end{aligned}
$$

where $a$ is given by Equation (45), $e$ is given by Equation (46) and $\varphi$ is given by the first equation of (58).

By neglecting the higher order terms of $e$, let the co-efficient of $\mu$ be denoted by $R$ then the complete Hamiltonian in terms of canonical variables $l, g, L, G$ is given by

$$
K=L\left[-2\left(n G+C_{0}\right)\right]^{\frac{1}{2}}-1+\mu R
$$

$\therefore$ The equations of motion for the complete Hamiltonian are

$$
\begin{aligned}
& \frac{\mathrm{d} L}{\mathrm{~d} \tau}=\frac{\mathrm{d} K}{\mathrm{~d} l}=\mu \frac{\partial R}{\partial l}, \quad \frac{\mathrm{d} G}{\mathrm{~d} \tau}=\frac{\mathrm{d} K}{\mathrm{~d} g}=\mu \frac{\partial R}{\partial g}, \\
& \frac{\mathrm{d} l}{\mathrm{~d} \tau}=-\frac{\mathrm{d} K}{\mathrm{~d} L}=-\left[-2\left(n G+C_{0}\right)\right]^{\frac{1}{2}}-\mu \frac{\partial R}{\partial L}, \\
& \frac{\mathrm{d} g}{\mathrm{~d} \tau}=-\frac{\mathrm{d} K}{\mathrm{~d} G}=\frac{n L}{\left[-2\left(n G+C_{0}\right)\right]^{\frac{1}{2}}}-\mu \frac{\partial R}{\partial G} .
\end{aligned}
$$

where

$$
\begin{aligned}
R= & 1-n(e L \sin l \sin 2 \varphi+G \cos 2 \varphi) \\
& -z\left[G+\frac{1}{r_{2}}+\frac{\varepsilon_{0}}{r_{3}}+\frac{A}{r_{2}^{3}}-\frac{B a^{2} n^{2}(1-2 e \cos l) \sin ^{2} 2 \varphi}{4 r_{2}^{5}}\right]
\end{aligned}
$$


The Equation (67) forms the basis of a general perturbation theory for the problem in question. The solution given in Equations ((62) and (63)) are periodic if $l$ and $g$ have commensurable frequencies that is, if

$$
\left|\frac{\eta_{l}}{\eta_{g}}\right|=\frac{2\left|n G+C_{0}\right|}{L}=\frac{p}{q}
$$

where $p$ and $q$ are integers.

The periods of $q_{i}, Q_{i}$ are $\frac{4 \pi}{\eta_{l}}$ and $\frac{4 \pi}{\eta_{g}}$, so that in case of commensurability, the period of the solution is $\frac{4 \pi p}{\eta_{l}}$ and $\frac{4 \pi q}{\eta_{g}}$.

\section{Existence of Periodic Orbits When $\mu \neq 0$}

Here we shall follow the method used by Choudhary [8] to prove the existence of periodic orbits when $\mu \neq 0$.

From Equations (67) when $\mu=0$, we have for

$$
\begin{aligned}
& \frac{\mathrm{d} L}{\mathrm{~d} \tau}=\frac{\partial K_{0}}{\partial l}=0, \quad \frac{\mathrm{d} G}{\mathrm{~d} \tau}=\frac{\partial G_{0}}{\partial g}=0, \\
& \frac{\mathrm{d} l}{\mathrm{~d} \tau}=-\frac{\partial K_{0}}{\partial L}=-\left[-2\left(n G+C_{0}\right)\right]^{\frac{1}{2}}=\eta_{1}(o) \quad \text { say } \\
& \frac{\mathrm{d} g}{\mathrm{~d} \tau}=-\frac{\partial K_{0}}{\partial G}=\frac{n L}{\left[-2\left(n G+C_{0}\right)\right]^{\frac{1}{2}}}=\eta_{2}(o) \quad \text { say }
\end{aligned}
$$

Let $x_{1}=L, x_{2}=G, y_{1}=l$ and $y_{2}=g$ then

$$
\frac{\mathrm{d} x_{1}}{\mathrm{~d} \tau}=\frac{\mathrm{d} x_{2}}{\mathrm{~d} \tau}=0, \quad \frac{\mathrm{d} y_{1}}{\mathrm{~d} \tau}=\eta_{1}(o), \quad \frac{\mathrm{d} y_{2}}{\mathrm{~d} \tau}=\eta_{2}(o)
$$

Thus the Equation (69) can be written as

$$
\begin{gathered}
\frac{\mathrm{d} x_{i^{\prime}}}{\mathrm{d} \tau}=0 \text { and } \frac{\mathrm{d} y_{i^{\prime}}}{\mathrm{d} \tau}=\eta_{i}(o) \\
\Rightarrow x_{i}=a_{i}, y_{i}=\eta_{i}(o) \tau+\omega_{i} \quad(i=1,2)
\end{gathered}
$$

These are generating solutions of the two-body problem.

Here $a_{i}, \eta_{i}$ are constants given by

$$
\eta_{1}(o)=\left[-\frac{\partial K_{0}}{\partial x_{1}}\right]_{x_{1}=a_{1}}, \quad \eta_{2}(o)=\left[-\frac{\partial K_{0}}{\partial x_{2}}\right]_{x_{2}=a_{2}}
$$

The generating solutions will be periodic with the period $\tau_{0}$ if

$$
x_{i}\left(\tau_{0}\right)-x_{i}(o)=0, \quad y_{i}\left(\tau_{0}\right)-y_{i}(o)=\eta_{i}(o) \tau=2 \pi \kappa_{i} \quad(i=1,2)
$$

Here $\kappa_{i}(i=1,2)$ are integers, so that $\eta_{i}(o)$ are commensurable.

Let the general solution in the neighbourhood of the generating solution be periodic with the period $\tau_{0}+\alpha \tau_{0}=(1+\alpha) \tau_{0}, \alpha$ is negligible quantity of the order of $\mu$. Let us introduce new independent variable $\varsigma$ by the equation 
$\varsigma=\frac{\tau}{1+\alpha}$. The period of the general solution will be

$\varsigma_{0}+\alpha \varsigma_{0}=(1+\alpha) \varsigma_{0}=(1+\alpha) \frac{\tau_{0}}{1+\alpha}=\tau_{0}$ which is same as the period of the generating solution. The Equation (67) now can be written as

$$
\frac{\mathrm{d} x_{i}}{\mathrm{~d} \varsigma}=(1+\alpha) \frac{\partial K}{\partial y_{i}}, \frac{\mathrm{d} y_{i}}{\mathrm{~d} \varsigma}=-(1+\alpha) \frac{\partial K}{\partial x_{i}}
$$

Following Poincare [9], the general solutions in the neighbourhood of the generating solutions may be written as

$$
x_{i}=a_{i}+\beta_{i}+q_{i}(\varsigma), \quad y_{i}=\eta_{i}(o) \varsigma+\omega_{i}+\gamma_{i}+\eta_{i}(\varsigma)
$$

Following the method of Hassan et al. [1], Bhatnagar [4] [5] and Choudhary [8], the Duboshin's conditions [2] for the existence of periodic orbits are given by

$$
\frac{\partial\left[K_{1}\right]}{\partial \omega_{i}}=0 \quad(i=1,2)
$$

$$
\begin{gathered}
\frac{\partial\left[K_{1}\right]}{\partial a_{i}}=0 \quad(i=1,2) \\
J=\frac{\partial\left(q_{2}, \eta_{1}, \eta_{2}\right)}{\partial\left(\gamma_{2}, \beta_{1}, \beta_{2}\right)} \neq 0 \quad\left(\text { with } \mu=\beta_{i}=\gamma_{i}=0\right)
\end{gathered}
$$

where $\left[K_{1}\right]$ is the first degree term of $K_{1}$ given in Equation (31).

Here the Equations ((75) and (76)) together justify the Equation (77).

Following Bhatnagar [5], Hassan et al. [1], Equation (77) can be written as

$$
\begin{gathered}
J=\left|\begin{array}{ccc}
\frac{\partial^{2}\left[K_{1}\right]}{\partial \omega_{2}^{2}} & 0 & 0 \\
\frac{\partial^{2}\left[K_{1}\right]}{\partial \omega_{2} \partial a_{1}} & -\tau_{0} \frac{\partial^{2} K_{0}}{\partial a_{1}^{2}} & -\tau_{0} \frac{\partial^{2} K_{0}}{\partial a_{2} \partial a_{1}} \\
\frac{\partial^{2}\left[K_{1}\right]}{\partial \omega_{2} \partial a_{2}} & -\tau_{0} \frac{\partial^{2} K_{0}}{\partial a_{1} \partial a_{2}} & -\tau_{0} \frac{\partial^{2} K_{0}}{\partial a_{2}^{2}}
\end{array}\right|=\tau_{0}^{2} \frac{\partial^{2}\left[K_{1}\right]}{\partial \omega_{2}^{2}}\left|\begin{array}{cc}
\frac{\partial^{2} K_{0}}{\partial a_{1}^{2}} & \frac{\partial^{2} K_{0}}{\partial a_{2} \partial a_{1}} \\
\frac{\partial^{2} K_{0}}{\partial a_{1} \partial a_{2}} & \frac{\partial^{2} K_{0}}{\partial a_{2}^{2}}
\end{array}\right| \\
\text { i.e., } J=\tau_{0}^{2} \frac{\partial^{2}\left[K_{1}\right]}{\partial \omega_{2}^{2}}\left[\frac{\partial^{2} K_{0}}{\partial a_{1}^{2}} \cdot \frac{\partial^{2} K_{0}}{\partial a_{2}^{2}}-\left(\frac{\partial^{2} K_{0}}{\partial a_{1} \partial a_{2}}\right)^{2}\right]
\end{gathered}
$$

From Equation (60),

$$
\begin{gathered}
K_{0}=a_{1}\left[-2\left(n a_{2}+C_{0}\right)\right]^{\frac{1}{2}}-1, \\
\frac{\partial K_{0}}{\partial a_{1}}=\left[-2\left(n a_{2}+C_{0}\right)\right]^{\frac{1}{2}}, \frac{\partial^{2} K_{0}}{\partial a_{1}^{2}}=0 \\
\text { and } \frac{\partial^{2} K_{0}}{\partial a_{1} \partial a_{2}}=\frac{-n}{\left[-2\left(n a_{2}+C_{0}\right)\right]^{\frac{1}{2}}} . \\
\therefore J=\frac{n^{2} \tau_{0}^{2}}{2\left(n a_{2}+C_{0}\right)} \cdot \frac{\partial^{2}\left[K_{1}\right]}{\partial \omega_{2}^{2}}
\end{gathered}
$$


Taking only zero degree terms (i.e., for $e=0, h=l=y_{1}$ )

$$
\begin{gathered}
r_{1}=n a=z, \quad r_{2}^{2}=1+n^{2} a^{2}+2 n a \cos 2 \varphi \\
r_{3}^{2}=1+n^{2} a^{2}+n a \cos 2 \varphi-\sqrt{3} n a \sin 2 \varphi=1+n^{2} a^{2}+2 n a \cos \left(2 \varphi+\frac{\pi}{3}\right), \\
2 \varphi=y_{1}+y_{2}-\frac{n \sqrt{x_{1}^{2}-x_{2}^{2}}}{2\left(n x_{2}+C_{0}\right)} \sin y_{1}, \\
y_{1}=\eta_{1}^{(o)} \varsigma+\omega_{1}+\gamma_{1}+\eta_{1}(\varsigma), \quad y_{2}=\eta_{2}^{(o)} \varsigma+\omega_{2}+\gamma_{2}+\eta_{2}(\varsigma), \\
x_{1}=a_{1}+\beta_{1}+\xi_{1}(\varsigma), \quad x_{2}=a_{2}+\beta_{2}+\xi_{2}(\varsigma), \\
\frac{\partial r_{2}}{\partial \omega_{i}}=-\frac{n a \sin 2 \varphi}{r_{2}}\left(\frac{2 \partial \varphi}{\partial \omega_{i}}\right), \quad \frac{\partial r_{3}}{\partial \omega_{i}}=-\frac{n a \sin \left(2 \varphi+\frac{\pi}{3}\right)}{r_{3}}\left(\frac{2 \partial \varphi}{\partial \omega_{i}}\right) \quad(i=1,2) . \\
\quad\left[K_{1}\right]=1-n G \cos 2 \varphi-n a\left[C_{1}+\frac{1}{r_{2}}+\frac{\varepsilon_{0}}{r_{3}}+\frac{A}{r_{2}^{3}}-\frac{B a^{2} n^{2} \sin ^{2} 2 \varphi}{4 r_{2}^{5}}\right] .
\end{gathered}
$$

Now,

$$
\begin{aligned}
& \frac{\partial\left[K_{1}\right]}{\partial \omega_{i}}=n G \sin 2 \varphi\left(2 \frac{\partial \varphi}{\partial \omega_{i}}\right)-n a\left[-\frac{1}{r_{2}^{2}} \frac{\partial r_{2}}{\partial \omega_{i}}-\frac{\varepsilon_{0}}{r_{3}^{2}} \frac{\partial r_{3}}{\partial \omega_{i}}-\frac{3 A}{r_{2}^{4}} \frac{\partial r_{2}}{\partial \omega_{i}}\right. \\
& \left.-\frac{B n^{2} a^{2}}{4}\left(\frac{r_{2}^{5} 2 \sin 2 \varphi \cdot \cos 2 \varphi 2 \frac{\partial \varphi}{\partial \omega_{i}}-\sin ^{2} 2 \varphi \cdot 5 r_{2}^{4} \frac{\partial r_{2}}{\partial \omega_{i}}}{r_{2}^{10}}\right)\right), \\
& =n G \sin 2 \varphi\left(2 \frac{\partial \varphi}{\partial \omega_{i}}\right)+\frac{n a}{r_{2}^{2}}\left(-\frac{n a \sin 2 \varphi}{r_{2}} 2 \frac{\partial \varphi}{\partial \omega_{i}}\right)+\frac{n a \varepsilon_{0}}{r_{3}^{2}}\left(-\frac{n a \sin \left(2 \varphi+\frac{\pi}{3}\right)}{r_{3}} 2 \frac{\partial \varphi}{\partial \omega_{i}}\right) \\
& +\frac{3 A n a}{r_{2}^{4}}\left(-\frac{n a \sin 2 \varphi}{r_{2}} 2 \frac{\partial \varphi}{\partial \omega_{i}}\right)+\frac{B n^{3} a^{3} \sin 2 \varphi \cdot \cos 2 \varphi}{2 r_{2}^{5}}\left(2 \frac{\partial \varphi}{\partial \omega_{i}}\right) \\
& -\frac{5 B n^{2} a^{2}}{4 r_{2}^{6}}\left(-\frac{n a \sin 2 \varphi}{r_{2}} 2 \frac{\partial \varphi}{\partial \omega_{i}}\right) \sin ^{2} 2 \varphi, \\
& =\left(2 \frac{\partial \varphi}{\partial \omega_{i}}\right)\left[n G \sin 2 \varphi-\frac{n^{2} a^{2} \sin 2 \varphi}{r_{2}^{3}}-\frac{n^{2} a^{2} \varepsilon_{0} \sin 2 \varphi}{2 r_{3}^{3}}-\frac{\sqrt{3} n^{2} a^{2} \varepsilon_{0} \cos 2 \varphi}{2 r_{3}^{3}}\right. \\
& \left.-\frac{3 A n^{2} a^{2} \sin 2 \varphi}{r_{2}^{5}}+\frac{B n^{3} a^{3} \sin 2 \varphi \cdot \cos 2 \varphi}{2 r_{2}^{5}}+\frac{5 B n^{3} a^{3} \sin ^{3} 2 \varphi}{4 r_{2}^{7}}\right], \\
& \frac{\partial\left[K_{1}\right]}{\partial \omega_{i}}=\left(2 \frac{\partial \varphi}{\partial \omega_{i}}\right)\left[\left(n G-\frac{n^{2} a^{2}}{r_{2}^{3}}-\frac{n^{2} a^{2} \varepsilon_{0}}{2 r_{3}^{3}}-\frac{3 A n^{2} a^{2}}{r_{2}^{5}}+\frac{5 B n^{4} a^{4} \sin ^{2} 2 \varphi}{4 r_{2}^{7}}\right) \sin 2 \varphi\right. \\
& \left.+\left(\frac{B n^{3} a^{3} \sin 2 \varphi}{2 r_{2}^{5}}-\frac{\sqrt{3} n^{2} a^{2} \varepsilon_{0}}{2 r_{3}^{3}}\right) \cos 2 \varphi\right] . \\
& \Rightarrow \frac{\partial\left[K_{1}\right]}{\partial \omega_{i}}=\left(2 \frac{\partial \varphi}{\partial \omega_{i}}\right) N, \quad \text { Similarly } \frac{\partial\left[K_{1}\right]}{\partial a_{i}}=\left(2 \frac{\partial \varphi}{\partial a_{i}}\right) N \quad(i=1,2)
\end{aligned}
$$

where 


$$
\begin{aligned}
N= & \left(n G-\frac{n^{2} a^{2}}{r_{2}^{3}}-\frac{n^{2} a^{2} \varepsilon_{0}}{2 r_{3}^{3}}-\frac{3 A n^{2} a^{2}}{r_{2}^{5}}+\frac{5 B n^{4} a^{4} \sin ^{2} 2 \varphi}{4 r_{2}^{7}}\right) \sin 2 \varphi \\
& +\left(\frac{B n^{3} a^{3} \sin 2 \varphi}{2 r_{2}^{5}}-\frac{\sqrt{3} n^{2} a^{2} \varepsilon_{0}}{2 r_{3}^{3}}\right) \cos 2 \varphi
\end{aligned}
$$

Here $\frac{\partial\left[K_{1}\right]}{\partial \omega_{i}}=\frac{\partial\left[K_{1}\right]}{\partial a_{i}}=0$ if and only if $N=0$ because $\frac{\partial \varphi}{\partial \omega_{i}}, \frac{\partial \varphi}{\partial a_{i}}(i=1,2)$ are not necessarily zero. For this, putting $\cos 2 \varphi=0$ then $\sin 2 \varphi=1$,

$$
\Rightarrow 2 \varphi=\frac{\pi}{2} \Rightarrow \varphi=\frac{\pi}{4}
$$

Thus from $N=0$, we get

$$
n G-\frac{n^{2} a^{2}}{r_{2}^{3}}-\frac{n^{2} a^{2} \varepsilon_{0}}{2 r_{3}^{3}}-\frac{3 A n^{2} a^{2}}{r_{2}^{5}}+\frac{5 B n^{4} a^{4}}{4 r_{2}^{7}}=0
$$

where $r_{2}^{2}=1+n^{2} a^{2}, r_{3}^{2}=1+n^{2} a^{2}-\sqrt{3} n a$.

From Equation (83),

$$
G=n^{2} a^{2}\left[\frac{1}{r_{2}^{3}}+\frac{\varepsilon_{0}}{2 r_{3}^{3}}+\frac{3 A}{r_{2}^{5}}-\frac{5 B n^{2} a^{2}}{4 r_{2}^{7}}\right],
$$

where $A$ and $B$ are given in Equation (31).

Now from Equation (81),

$$
\begin{gathered}
\frac{\partial\left[K_{1}\right]}{\partial \omega_{2}}=2 \frac{\partial \varphi}{\partial \omega_{2}} N \\
\frac{\partial^{2}\left[K_{1}\right]}{\partial \omega_{2}^{2}}=2\left[\frac{\partial^{2} \varphi}{\partial \omega_{2}^{2}} N+\frac{\partial \varphi}{\partial \omega_{2}} \frac{\partial N}{\partial \omega_{2}}\right] \\
2 \varphi=y_{1}+y_{2}-\frac{n \sqrt{x_{1}^{2}-x_{2}^{2}}}{2\left(n x_{2}+C_{0}\right)} \sin y_{1} \\
2 \frac{\partial \varphi}{\partial \omega_{1}}=1-\frac{n \sqrt{x_{1}^{2}-x_{2}^{2}}}{2\left(n x_{2}+C_{0}\right)} \cos y_{1} \\
2 \frac{\partial \varphi}{\partial \omega_{2}}=1 \quad \Rightarrow 2 \frac{\partial^{2} \varphi}{\partial \omega_{2}^{2}}=0 \\
\Rightarrow \frac{\partial^{2}\left[K_{1}\right]}{\partial \omega_{2}^{2}}=\frac{\partial N}{\partial \omega_{2}},
\end{gathered}
$$

where

$$
\begin{aligned}
N= & \left(n G-\frac{n^{2} a^{2}}{r_{2}^{3}}-\frac{n^{2} a^{2} \varepsilon_{0}}{2 r_{3}^{3}}-\frac{3 A n^{2} a^{2}}{r_{2}^{5}}\right) \sin 2 \varphi+\frac{5 B n^{4} a^{4}}{4}\left(\frac{\sin ^{3} 2 \varphi}{r_{2}^{7}}\right) \\
& +\frac{B n^{3} a^{3}}{4}\left(\frac{\sin 4 \varphi}{r_{2}^{5}}\right)-\frac{\sqrt{3} n^{2} a^{2} \varepsilon_{0}}{2}\left(\frac{\cos 2 \varphi}{r_{3}^{3}}\right)
\end{aligned}
$$

Differentiating $N$ partially with respect to $\omega_{2}$, we get

$$
\begin{aligned}
\frac{\partial N}{\partial \omega_{2}}= & -\frac{3 n^{3} a^{3}}{2 r_{2}^{5}}\left(2+\sigma_{1}-\sigma_{2}\right)+\frac{5 n^{3} a^{3}}{4 r_{2}^{9}}\left[3 n^{2} a^{2}\left(3 \sigma_{1}-5 \sigma_{2}\right)-\frac{6}{\pi}\left(2 \sigma_{1}-\sigma_{2}\right)\right] \\
& -\frac{\sqrt{3} n^{3} a^{3} \varepsilon_{0}}{4 r_{3}^{5}}\left[3 \sqrt{3} n a-2-2 n^{2} a^{2}\right]
\end{aligned}
$$




$$
\begin{aligned}
\frac{\partial N}{\partial \omega_{2}}= & -\frac{3 n^{3} a^{3}}{2 r_{2}^{5}}\left(2+\sigma_{1}-\sigma_{2}\right)-\frac{5 n^{3} a^{3}}{4 r_{2}^{9}}\left[6\left(2 \sigma_{1}-\sigma_{2}\right)-3 n^{2} a^{2}\left(3 \sigma_{1}-5 \sigma_{2}\right)\right] \\
& +\frac{\sqrt{3} n^{2} a^{2} \varepsilon_{0}}{4 r_{3}^{5}}\left[2+2 n^{2} a^{2}-3 \sqrt{3} n a\right]
\end{aligned}
$$

where $\cos 2 \varphi=0, \sin 2 \varphi=1$.

If the collaboration of Equations ((83) and (84)) gives $\frac{\partial N}{\partial \omega_{2}} \neq 0$ and then $\frac{\partial^{2}\left[K_{1}\right]}{\partial \omega_{2}^{2}} \neq 0$ which implies $J \neq 0$ i.e., the orbit of the infinitesimal mass is periodic. This condition of periodic orbits given by Duboshin [2] may be confirmed by the regular trajectory of the Poincare surface of sections as in Hassan et al. [1] with suitable values of the parameters of $\mu, \sigma_{1}, \sigma_{2}$.

\section{Discussions and Conclusions}

In order to prove the existence of periodic orbits of the first kind in the CR4BP, we have discussed the problem into five sections starting with introduction about the historical evolution of the topic. In the second section, we established the equations of motion of the infinitesimal mass under the perturbed gravitational field of the three primaries. In the present problem, the second primary is a tri-axial rigid body and other two are point masses i.e., spheres. All the primaries are moving on their own circular orbits about the centre of mass of the dominant primaries $P_{1}$ and $P_{2}$. The primaries $P_{1}$ and $P_{2}$ are dominant in the sense that $P_{1}$ and $P_{2}$ have influence of attraction on the third primary $P_{3}$ and infinitesimal mass $P_{4}$ but $P_{3}$ and $P_{4}$ have no influence of attraction on the primaries $P_{1}$ and $P_{2}$ whereas $P_{3}$ has an influence of attraction on the infinitesimal mass $P_{4}$ only but not on $P_{1}$ and $P_{2}$. That's the reason for which the centre of mass of $P_{1}$ and $P_{2}$ didn't change. The second section ended with the energy integral of the infinitesimal mass at $P_{4}\left(x_{1}, x_{2}\right)$.

The energy function $H$ contains three singularities $r_{1}=0, r_{2}=0$ and $r_{3}=0$ so in Hamiltonian mechanics, to keep the energy function $H=$ constant, we need to eliminate any singularity for the case of collision with the corresponding primary. In the third section, we have introduced a suitable generating function for regularization of $H$ to eliminate the singularity at $r_{1}=0$. After regularizing the Hamiltonian $H=C$, we have developed the canonical equations of motion corresponding to the regularized Hamiltonian $K=0$.

In fourth section, we have established the generating solution i.e., the solutions of the equations of motion of the infinitesimal mass by taking the first primary at the origin i.e., at the centre of mass. On this consideration, we get $\mu=0$ and the Hamiltonian becomes $K_{0}$. By taking $K_{0}$ as our Hamiltonian, we get the solution of the equations of motion, which is called generating solution. With the help of generating solution and the method of analytic continuation, we can find the general solution corresponding to the complete Hamiltonian $K=K_{0}+\mu K_{1}$ where $\mu \neq 0$. 
In fifth section, we have examined the existence of periodic orbits when $\mu \neq 0$ with the technique of Choudhary [8] applying to the conditions given by Duboshin [2]. Since our consideration satisfied all the conditions for periodic orbits given by Duboshin [2], hence we conclude that the periodic orbits of the infinitesimal mass around the first primary exist when suitable values of $\mu, \sigma_{1}, \sigma_{2}$ are taken. By shifting the origin to the centre of the other primaries also, the existence of periodic orbits can be examined. Even by using "Mathematica", we can show the existence of periodic orbits of the infinitesimal mass around other primaries also, by taking suitable values of the parameters.

\section{References}

[1] Hassan, M.R., Hassan, Md. A., Singh, P., Kumar, V. and Thapa, R.R. (2017) Existence of Periodic Orbits of the First Kind in the Autonomous Four-Body Problem with the Case of Collision. International Journal of Astronomy and Astrophysics, 7, 91-111. https://doi.org/10.4236/ijaa.2017.72008

[2] Duboshin, G.N. (1964) Analytical and Qualitative Methods (Russian). Celestial Mechanics, 178-184.

[3] Giacaglia, E.O. (1967) Periodic Orbits of Collision in the Restricted Problem of Three Bodies. Astronomical Journal, 72, 386-391. https://doi.org/10.1086/110237

[4] Bhatnagar, K.B. (1969) Periodic Orbits of Collision in the Plane Elliptic Restricted Problem of Three Bodies. National Institute of Science, 35A, 829-844.

[5] Bhatnagar, K.B. (1971) Periodic Orbits of Collision in the Plane Circular Problem of Four Bodies. Indian Journal of Pure and Applied Mathematics, 2, 583-596.

[6] Ceccaroni, M. and Biggs, J. (2012) Low-Thrust Propulsion in a Coplanar Circular Restricted Four Body Problem. Celestial Mechanics and Dynamical Astronomy, 112, 191-219. https://doi.org/10.1007/s10569-011-9391-X

[7] McCuskey, C.W. (1963) Introduction to Celestial Mechanics. Addison-Wesley, Pearson Education, Massachusetts, USA.

[8] Choudhry, R.K. (1966) Existence of Periodic Orbits of the Third kind in the Elliptic Restricted Problem of the Three Bodies and the Stability of the Generating Solution. Proceedings of the National Academy of Sciences, 36A, 249-264.

[9] Poincare, H. (1905) Lecons de Mécanique Céleste. Gauthier-Villars, Paris, 1. 
Submit or recommend next manuscript to SCIRP and we will provide best service for you:

Accepting pre-submission inquiries through Email, Facebook, LinkedIn, Twitter, etc. A wide selection of journals (inclusive of 9 subjects, more than 200 journals)

Providing 24-hour high-quality service

User-friendly online submission system

Fair and swift peer-review system

Efficient typesetting and proofreading procedure

Display of the result of downloads and visits, as well as the number of cited articles Maximum dissemination of your research work

Submit your manuscript at: http://papersubmission.scirp.org/

Or contact ijaa@scirp.org 